\title{
Enfrentamento e Adaptação de Pacientes na Amputação por Trauma ou Doença
}

\section{Patients Coping and Adaptation in the Amputation by Trauma or Disease}

\author{
Ana Paula da Silva Vasques Oliveira ${ }^{1}$ \\ Fabrício Fernandes Almeida ${ }^{2}$
}

\section{RESUMO}

Introdução: A amputação é uma perda física e psíquica. A pessoa amputada precisa se adaptar a nova forma de vida. Objetivo: O presente estudo teve como objetivo avaliar o impacto da estratégia de enfrentamento no processo de adaptação de pacientes amputados devido a algum trauma (acidente) ou doença. Método: Foram aplicados entrevista semiestruturada, Escala Modo de Enfrentamento de Problemas - EMEP e questionário sociodemográfico no período de agosto a outubro de 2015, no Hospital de Base do Distrito Federal. Realizou-se um estudo quantitativo de corte transversal, com amostra não probabilística por conveniência. Os critérios de inclusão da pesquisa foram todos os pacientes que sofreram amputação por trauma ou doença, com condições cognitivas de participar da pesquisa e que aceitaram participar. Resultados: Dos 31 entrevistados, 9,7\% sofreram amputação por trauma e 90,3\% por doença. Foram considerados mal adaptados ao tratamento $12,9 \%$ dos entrevistados. A amostra encontrada foi dividida em dois grupos em relação ao seu modo de enfrentamento, a saber: a) enfrentamento focalizado no problema e b) práticas religiosas/pensamento fantasioso. Conclusão: $\mathrm{A}$ análise dos achados sugere uma não associação entre estratégias de enfrentamento e adaptação, dada a não variação nas estratégias de enfrentamento encontradas. Não foi possível concluir se não existe diferença quanto à estratégia de enfrentamento de pacientes amputados por trauma ou por doença.

DESCRITORES: Enfrentamento. Amputação. Psicologia. Hospital.

\begin{abstract}
Introduction: Amputation is a physical and psychological loss. The amputated person needs to adapt to a new way of life. Objective: The present study aimed to evaluate the coping strategy impact into the adaptation process of the amputated patient due to trauma (accident) or disease. Methods: Semi-structured interview, Ways of Coping Scale and sociodemographic questionnaire were applied during August, September and October of 2015 in the "Hospital de Base of Distrito Federal" and performing a quantitative cross-sectional study, with a non-probabilistic sample by convenience. The inclusion criteria of the research were all of the patients who suffered amputation due to trauma or disease, with cognitive conditions to participate in the research and who accepted to participate. Results: Among the 31 participants, $9,7 \%$ suffered amputation due to trauma and $90.3 \%$ due to a disease. Only $12.9 \%$ of the participants were considered ill-adapted to the treatment. The sample found was divided into two groups in relation to their mode of coping, namely: a) problem-focused and b) religious practices/fantasy thinking. Conclusion: The analysis of the findings suggests no association between coping strategies and adaptation, given the non-variation in coping strategies encountered. It was not possible to conclude that there is no difference in the coping strategy of patients amputated due to trauma or illness.
\end{abstract}

DESCRIPTORS: Coping. Amputation. Psychology. Hospital.

1- Psicóloga Residente em psicologia hospitalar no Hospital de Base do Distrito Federal - HBDF. Brasília. DF. Brasil.

2- Psicólogo e Preceptor no Hospital de Base do Distrito Federal (HBDF) da Secretaria de Estado de Saúde (SES/DF). Brasília. DF. Brasil. 
A amputação consiste em uma cirurgia, na qual se separa, parcial ou totalmente, um membro ou outra parte do corpo ${ }^{1}$. O processo de amputação não deve ser visto como uma mutilação do corpo, mas uma busca da recuperação de sua saúde, sendo que a prótese pode contribuir para uma melhora na qualidade de vida e interação com a sociedade ${ }^{2}$.

Apesar de o método parecer uma agressão é realizado para restabelecer a saúde dos pacientes, eliminando o membro infectado que não pode ser recuperado, sem que haja contaminação dos outros membros, por isso, trata-se de um procedimento eficiente ${ }^{2}$.

A reabilitação deve oferecer ao paciente um existir modificado, mas que seja possível ${ }^{3}$. A amputação pode trazer consequências físicas e psicológicas, afetando o paciente e sua família, sendo mais aceita quando vista como um modo de prolongar a existência, dar alívio à dor e trazer a possibilidade do fim da internação ${ }^{4}$. O psicólogo hospitalar pode contribuir significativamente para a reabilitação dos pacientes, auxiliando-os na busca de estratégias de enfrentamento adaptativas.

Um estudo realizado no Hospital Universitário de Santa Maria no período de 2002 a 2003, com 154 sujeitos, concluiu que $67,5 \%$ das amputações tiveram como causa a enfermidade vascular e/ou infecciosa, $17,5 \%$ causas traumáticas, $12,4 \%$ causas desconhecidas, $1,3 \%$ causas congênitas e $1,3 \%$ causas tumorais. Em relação ao sexo, 74,6\% dos pacientes eram do sexo masculino ${ }^{5}$.

Outro estudo aponta que a hospitalização favoreceu para que os entrevistados refletissem sobre o modo de vida e se propusesse a fazer mudanças, aumentando o autocuidado, evitando o uso de cigarro e bebida e alimentando-se de forma saudável ${ }^{6}$. O tabagismo está entre as comorbidades mais prevalentes nos casos de amputação devido a doenças vasculares $(59 \%)^{7}$.

Traumas relacionados a acidentes de trânsito e de trabalho, atropelamentos, doenças ateroscleróticas, moléstias tropicais e doenças como o diabetes, são as causas principais das amputações ${ }^{1}$. Uma pesquisa realizada com 11 pacientes amputados em dois hospitais públicos de Porto Alegre, no ano de 2009, teve como amputação mais frequente os dedos do pé (46\%), seguido pelo antepé $(18 \%)$ e pé $(18 \%)^{8}$.

O paciente que amputou a perna sentirá a perna fantasma como um membro real e permanecerá aberto as ações das quais ela é capaz de fazer, podendo cair ao tentar caminhar ${ }^{9}$. Mesmo quando o membro foi retirado, o cérebro emite sensações ${ }^{2}$ Para alguns pacientes o membro amputado se apresenta como deformado, contorcido ou desproporcional.

Após a amputação muitas pessoas deparam-se com um sentimento de estranhamento de si, não reconhecendo suas próprias reações emocionais ou até a si mesmas fisicamente ${ }^{10}$. A debilidade física, as novas limitações, a dor e a angústia resultantes da situação de dependência aumentam o estresse do indivíduo internado. Em alguns casos existe a necessidade da modificação de sua rotina e do domínio de sua vida ${ }^{11}$.

O tratamento do portador de doença crônica deve favorecer a adaptação a essa condição, pois é preciso que o paciente seja capaz de conviver com a mudança imposta ${ }^{12}$. A relação do paciente com a hospitalização depende dos recursos da instituição e do repertorio de recursos pessoais que os pacientes trazem de suas vivências. Esses recursos auxiliam ou prejudicam a relação com a instituição e interfere no modo de enfrentamento da doença ${ }^{3}$.

O enfrentamento é considerado como qualquer tentativa de preservar a saúde mental e física, sendo uma série de respostas que envolvem a interação do indivíduo com o ambiente, porém nem todas as estratégias de enfrentamento são igualmente eficazes. Algumas proporcionam alívio temporário em curto prazo, sendo mal adaptativas a longo prazo $^{13}$. O termo enfrentamento tem sido empregado em psicologia da saúde referindo-se a forma como o indivíduo administra uma situação adversa ou estressora ${ }^{14}$.

Um estudo sobre o processo de enfrentamento no pós-tratamento do câncer de mama concluiu que as estratégias mais empregadas pelos sujeitos foram as focalizadas no problema ${ }^{15}$. Outro estudo apontou que os sujeitos que receberam preparo psicológico utilizaram maior número de estratégias de enfrentamento adaptativos ${ }^{16}$.

Este trabalho objetivou, então, avaliar o impacto da estratégia de enfrentamento no processo de adaptação de pacientes do Hospital de Base do Distrito Federal. Esta pesquisa pode trazer contribuições ao colaborar com a ampliação teórica a esse respeito e auxiliar na qualidade de vida de pacientes amputados uma vez que o procedimento de amputação é bastante frequente nos hospitais e tem o potencial de interferir diretamente no processo psíquico dos pacientes e seus familiares.

Esse estudo pode auxiliar o profissional psicólogo no preparo pré-cirúrgico e pós-cirúrgico de pacientes e seus familiares, além de favorecer uma maior proximidade com pacientes amputa- 
dos, possibilitando uma melhor compreensão e reflexão para a equipe, em relação aos próprios comportamentos.

\section{MÉTODO}

Este estudo quantitativo de corte transversal, com amostra não probabilística por conveniência foi aprovado pelo Comitê de Ética da Fundação de Ensino e Pesquisa em Ciências da Saúde da Secretaria do Estado de Saúde do Distrito Federal mediante o CAAE: 47377315.2.0000.5553, recebendo parecer favorável sob o n ${ }^{\circ}$ 1.196.683. Aanálise dos resultados foi realizada com o auxílio do programa Statistical Package for Social Sciences (SPSS) 20.0. Para a avaliação de associação entre as variáveis, utilizou-se do cálculo não paramétrico dos valores de dispersão, qui-quadrado $\left(\mathrm{X}^{2}\right)$ e do Teste Exato de Fisher, respeitando-se os graus de liberdade condizentes com as variáveis nominais investigadas, para a correção das comparações de amostra com baixo número de sujeitos, ambos com um alfa de $95 \%$.

Os dados foram coletados por meio do questionário sociodemográfico, prontuário dos pacientes, Escala Modo de Enfrentamento de Problemas (EMEP) e entrevista clínica e diagnóstica, aplicados nos meses agosto, setembro e outubro do ano de 2015, em pacientes amputados no Hospital de Base do Distrito Federal (HBDF), hospital terciário de alta complexidade, que atende aos usuários do Sistema Único de Saúde (SUS).

A população desse estudo foi composta por 31 pacientes submetidos à amputação de membros superiores, inferiores ou tronco, mais especificamente, os seios. O critério de inclusão da pesquisa foram todos os pacientes que sofreram amputação por trauma ou doença, com condições cognitivas de participar da pesquisa, que aceitaram participar e que estavam disponíveis durante o período de coleta de dados.

O Termo de consentimento Livre e Esclarecido foi apresentado juntamente com a aplicação do questionário sociodemográfico, da entrevista semiestruturada e da Escala Modo de Enfrentamento de Problemas (EMEP) durante a hospitalização do paciente, no pós-operatório, em seu próprio leito. A aplicação foi assistida, a pesquisadora lia o instrumento e assinalava a opção informada pelos pacientes.

O questionário sociodemográfico foi utilizado para a caracterização dos pacientes, onde coletou-se os seguintes dados: sexo, idade, procedência, naturalidade, estado civil, grau de escolaridade, religião, profissão, o local da amputação e tipo de amputação (amputação por doença ou amputação por trauma). Foram feitas quatro perguntas com duas opções de resposta (Sim ou Não): 1) Alguém da família tem/teve a mesma doença?; 2) Possui filhos?; 3) Recebeu preparo pré-cirúrgico?; 4) A família/amigos participou do processo cirúrgico?. Ocorreu também a verificação do suporte social.

A avaliação do suporte social foi dividida em suporte operacional, que consiste na disponibilização de ajuda para a realização de atividades do cotidiano, auxílio material e financeiro ao paciente, e em suporte emocional, que se refere à disponibilização de familiares e/ou amigos para escutar ou fazer companhia ao paciente, fazendo com que se sinta cuidado ${ }^{17}$. Os entrevistados puderam classificar o suporte operacional como funcional ou disfuncional e o suporte emocional como satisfatório ou insatisfatório.

Os laços sociais e os relacionamentos com outras pessoas nos afetam de forma positiva ou negativa. Pessoas que recebem um nível elevado de apoio social podem experimentar menos estresse e enfrentá-lo de forma mais eficaz ao vivenciar uma situação estressante ${ }^{13}$. Porém, existem casos em que o apoio social não reduz o estresse, como nos casos em que o apoio oferecido não é o adequado para o momento ou quando a pessoa não deseja o auxílio.

A entrevista semiestruturada buscou avaliar se houve mudanças na vida do paciente após a amputação e foi baseada nos critérios diagnósticos para Transtornos de Adaptação ${ }^{18}$, com o objetivo de caracterizar o paciente como adaptado ou não adaptado após o procedimento cirúrgico.

Foram considerados como mal adaptados os entrevistados que apresentaram sofrimento e perturbação emocional com prejuízo no funcionamento e desempenho sociais e com no mínimo três dos seguintes sintomas: humor deprimido; ansiedade; preocupação; sentimento de incapacidade de adaptação e/ou, planejar o futuro e/ou continuar na situação atual; e qualquer grau de incompetência no desempenho da rotina diária ${ }^{18}$.

A Escala Modo de Enfrentamento de Problemas (EMEP) foi uma adaptação por Gimenes, Queiroz e Favero da escala construída por Vitaliano e colaboradores, tendo sido validada para a população brasileira ${ }^{19}$. Trata-se de um questionário composto por 45 itens, que incluem pensamentos e ações utilizados pelas pessoas para lidar com demandas internas e externas de um evento estressante. A EMEP é respondida por meio de 
uma escala likert de cinco pontos (1 - eu nunca faço isso; 2 - eu faço isso um pouco; 3 - eu faço isso ás vezes; 4 - eu faço isso muito; 5 - eu faço isso sempre).

A escala conceitua enfrentamento como um conjunto de respostas específicas para uma dada situação estressora ${ }^{19}$. A versão brasileira identifica quatro modos de enfrentamento: 1) Estratégias de enfrentamento focalizadas no problema; 2) Estratégias de enfrentamento focalizadas na emoção; 3) Práticas religiosas/pensamento fantasioso e 4) Busca de suporte social. Os escores da EMEP são calculados para cada fator e o resultado baseia-se na comparação das médias obtidas em cada fator ${ }^{14}$.

A estratégia de enfrentamento focalizada no problema é composta por dezoito itens que englobam estratégias de aproximação em relação ao estressor, no sentido de solucionar o problema, lidar ou manejar a situação estressora e inclui itens que envolvem estratégias cognitivas voltadas para a reavaliação positiva do problema ${ }^{14}$.

A estratégia de enfrentamento focalizada na emoção é composta por quinze itens que abarcam pensamento irrealista, esquiva, negação, expressão de raiva, tensão e reações de culpabilização de outra pessoa ou si próprio ${ }^{14}$.

No comportamento focalizado na emoção a pessoa tenta controlar a resposta emocional ao estressor utilizando estratégias comportamentais e cognitivas. As estratégias comportamentais incluem a busca de apoio social, uso de álcool ou drogas ou ocupar-se para desviar a atenção do problema. As cognitivas envolvem mudar o modo de avaliar o estressor ou negar as informações desagradáveis. As pessoas geralmente utilizam o enfretamento focalizado na emoção quando acreditam que pouco ou nada podem fazer para alterar a situação estressante ou que seus recursos ou capacidades de enfrentamento são insuficientes ${ }^{13}$.
A estratégia de enfrentamento focalizada nas práticas religiosas e pensamento fantasioso é composta por sete itens que envolvem comportamentos e pensamentos religiosos e/ou fantasiosos como auxilio no enfrentamento do problema.

A estratégia focalizada na busca de suporte social é composta por cinco itens que representam a busca de apoio instrumental, emocional ou de informação para auxiliar no enfrentamento da situação ${ }^{14}$.

\section{RESULTADOS}

A amostra encontrada foi dividida em dois grandes grupos em relação ao seu modo de enfrentamento, a saber: a) enfrentamento focalizado no problema, representando $48,4 \%$ da amostra; e b) práticas religiosas/pensamento fantasioso, representando $48,4 \%$ da amostra. A estratégia de enfrentamento focalizada na busca por suporte social apresentou-se majoritária para um único sujeito (Tabela 1 ).

Referente ao motivo por ter ocorrido à amputação 9,7\% dos pacientes sofreram amputação por trauma e $90,3 \%$ por doença. Dos 31 sujeitos do estudo treze tinham o diagnóstico de diabetes, dez de doenças obstrutivas, quatro de câncer e um tinha o diagnóstico de aneurisma. Em relação às amputações por trauma dois dos entrevistados sofreram perfuração por arma de fogo (PAF) e um sofreu um atropelamento (Figura 1).

O sexo masculino foi mais frequente, (21 masculino e 10 feminino). A faixa etária foi de 18 a 86 anos, sendo a média de idade 56,81 anos. Das áreas amputadas $77,4 \%$ correspondiam a membros inferiores incompletos, $9,7 \%$ as regiões do tronco, $6,5 \%$ a membros superiores incompletos e 3,2\% a membros inferiores completos e membros superiores completos, respectivamente. Todos os pacientes avaliados como mal adaptados sofreram

Tabela 1: Estratégias de Enfrentamento adotadas pelos 31 entrevistados. Coleta realizada no Hospital de Base do DF entre agosto a outubro de 2015.

\begin{tabular}{l|c|c|c|c}
\hline & Frequência & Porcentagem & $\begin{array}{c}\text { Porcentagem } \\
\text { válida }\end{array}$ & $\begin{array}{c}\text { Porcentagem } \\
\text { acumulativa }\end{array}$ \\
\hline Problema & 15 & 48.4 & 48.4 & 48.4 \\
Fantasioso & 15 & 48.4 & 48.4 & 96.8 \\
Social & 1 & 3.2 & 3.2 & 100.0 \\
Total & 31 & 100.0 & 100.0 & \\
\hline
\end{tabular}


Figura 1: Tipo de adoecimento dos entrevistados. Coleta realizada no Hospital de Base do DF entre Agosto a Outubro de 2015.

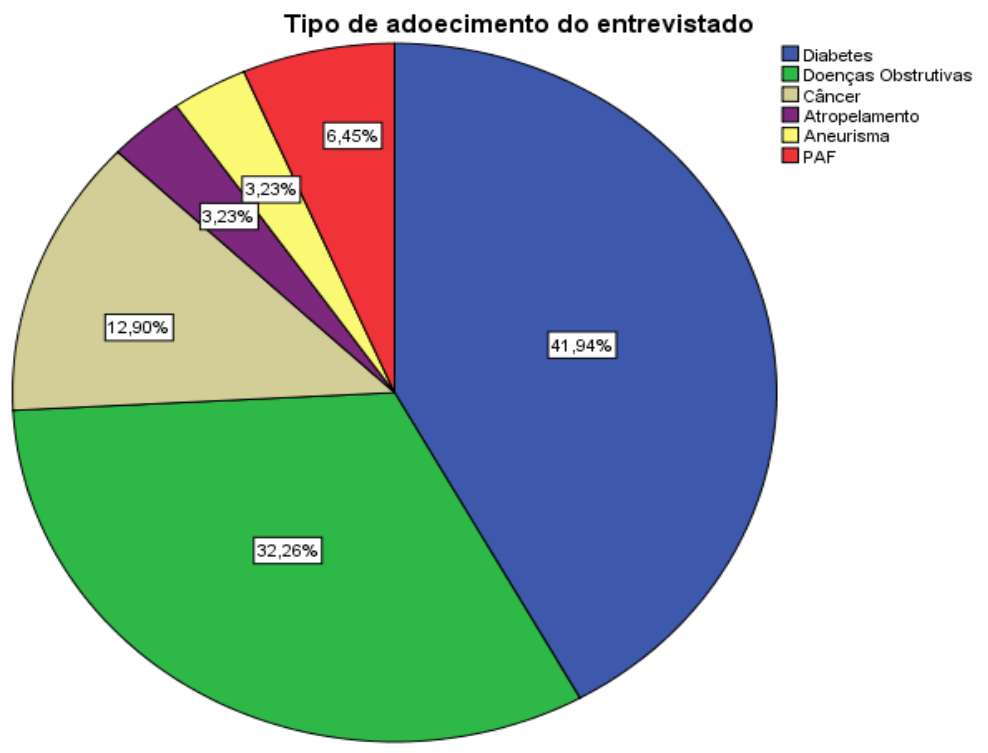

amputação nos membros inferiores incompletos. 0 procedimento cirúrgico de amputação estava sendo realizado pela segunda vez por seis pacientes.

Dos entrevistados, 17 eram tabagistas ou ex-tabagistas, sendo que todos que foram diagnosticados por doenças obstrutivas tinham histórico prévio ou anterior de uso de tabaco. Quanto ao acompanhamento psicológico, apenas cinco dos pacientes estavam sendo acompanhados pela psicologia. Em relação à religião 18 eram católicos, 12 evangélicos e um não declarou vinculação religiosa.

A naturalidade prevalente foi Minas Gerais $(25,8 \%)$ e o local de moradia Goiás $(25,8 \%)$. No Distrito Federal a região mais frequente foi Ceilândia (12,9\%). Quanto ao estado civil, 11 dos entrevistados estavam solteiros, seguidos de nove casados, quatro divorciados, quatro em união estável e três viúvos. Houve grande dispersão dos dados relacionados à profissão dos entrevistados, impossibilitando sua categorização, mas as que tiveram maior prevalência foram: domésticas $(12,9 \%)$ e, respectivamente, $6,5 \%$ para cozinheiras e trabalhadores rurais.

Em relação à escolaridade 17 cursaram o primeiro grau incompleto, cinco tinham o segundo grau completo, quatro eram analfabetos e quatro possuíam o primeiro grau completo, apenas um cursou o nível superior.

Dos entrevistados, 25 tinham filhos. Em relação à avaliação do pesquisador quanto à adaptação, 27 pacientes foram avaliados como bem adaptados e quatro como mal adaptados. Todos pacientes que sofreram amputação por trauma não receberam preparo pré-cirúrgico e dos que amputaram por doença cinco também não receberam esse preparo.

A maioria dos pacientes decidiu pela amputação, apenas $25,8 \%$ não puderam autorizar devido à impossibilidade momentânea, quando acordaram já estavam sem o membro. Quanto ao apoio social, 28 pacientes afirmaram sentirem-se apoiados pela família/amigos e três avaliaram que não receberam apoio. Em relação ao suporte operacional e emocional 22 dos entrevistados afirmaram satisfação, nove estavam insatisfeitos.

Os entrevistados relataram a ocorrência de mudanças em sua vida social, profissional e familiar após a amputação, para 12 houve um aumento da dependência. Referente aos sintomas emocionais, 14 afirmaram o sentimento de tristeza.

Quando questionados sobre o que estariam fazendo, caso não existisse motivos para que a amputação ocorresse, 21 dos entrevistados afir- 
maram que estariam trabalhando. Além da amputação, alguns dos entrevistados estavam vivendo outros fatores de estresse como luto, desemprego, prisão, nascimento de filho, abandono familiar e descoberta de diagnóstico adicional.

Grande parte dos entrevistados avaliou ser a amputação algo positivo, devido às dores intensas que sentiam. Sendo a retirada do órgão uma possibilidade de adiar a morte. Porém, os sentimentos se mostraram ambivalentes e contraditórios, sendo possível identificar tristeza, vergonha, culpa, esperança, gratidão e revolta.

As estratégias de enfrentamento mais utilizadas em outro estudo foram as focalizadas no problema e na emoção. De acordo com os autores estas estratégias têm um poder mais preditivo para a resolução do caso $^{14}$. Consequentemente os enfrentamentos focalizados nas práticas religiosas/ pensamento fantasioso e na busca de suporte social podem ser considerados como enfrentamentos acessórios. $\mathrm{Na}$ amostra deste estudo 100\% dos sujeitos tiveram enfrentamentos primários focalizados no problema o que inviabiliza a análise do impacto do modo de enfrentamento no tipo de adaptação e nas demais variáveis estudadas.

Para o cálculo da EMEP, então, foi traçada uma estratégia alternativa ${ }^{14}$. Os valores encontrados na EMEP não foram divididos em primários e secundários, mas transformados em valores absolutos percentuais, assim, foi obtido valor de qual é o fator predominante majoritário em formato percentual. Para tanto, foi realizado um cálculo de percentil (valor do fator x $100 \div$ pelo total do fator). Devido a EMEP ter um número diferente de questões por fator, todos foram padronizados em percentual e posteriormente foi calculado qual o enfrentamento tinha um maior percentual por pessoa.

Os dados não demonstram associação entre estratégias de enfrentamento e a adaptação, $\mathrm{X}^{2}$. $=1,340, \mathrm{GL}=2$ e $\alpha=0,512$. Porém, dada $a$ não variação nas estratégias de enfrentamento na emoção e no problema e o n da amostra ser pequeno, não foi possível realizar afirmações a respeito do comportamento de associações que envolvam esta variável.

No que se refere à análise do impacto entre preparo prévio para a cirurgia e estratégia de enfrentamento utilizada, a associação destas variáveis apresentou $X^{2}=1,056, G L=2$ e $\alpha=0,590$ e em relação entre preparo prévio e à adaptação $\mathrm{X}^{2}=1,404, \mathrm{GL}=1$ e $\alpha=0,236$ com Teste Exato de Fisher $=0,268$, não demonstrando uma associação entre essas variáveis.

Referente ao impacto entre os pacientes acompanhados pela psicologia e a estratégia de enfrentamento utilizada, a associação destas variáveis apresentou $\mathrm{X}^{2}=0,445 \mathrm{GL}=2$ e $\alpha=0,800$. Quanto aos pacientes acompanhados pela psicologia e a adaptação, $\mathrm{X}^{2}=0,883 \mathrm{GL}=1$ e $\alpha=0,347$ com Teste Exato de Fisher =1,00, evidenciando uma não associação dos dados.

Também não houve associação dos dados entre o fato de o entrevistado ter filhos e a estratégia de enfrentamento utilizada, tendo a associação dessas variáveis apresentado $\mathrm{X}^{2}=0,248 \mathrm{GL}=2$ e $\alpha=0,883$, assim como entre o sexo dos entrevistados e a estratégia de enfrentamento utilizada, apresentando $X^{2}=0,492, G L=2$ e $\alpha=0,782$.

Não houve associação entre o tipo de amputação e o enfrentamento majoritário. A associação dessas variáveis apresentou $X^{2}=0,492$ $\mathrm{GL}=2$ e $\alpha=0,782$, e entre o tipo de amputação e a adaptação, $X^{2}=1,234 \mathrm{GL}=1$ e $\alpha=0,267 \mathrm{com}$ Teste Exato de Fisher $=0,349$. Não foi possível comparar a relação entre a área amputada e o enfrentamento e a adaptação, pois houve uma prevalência nos membros inferiores incompleto.

A associação das variáveis religião e enfrentamento majoritário apresentou $X^{2}=2,985$ $\mathrm{GL}=4$ e $\alpha=0,560$. Houve mais católicos com a estratégia de enfrentamento focalizada no problema, se comparados com evangélicos, dentre os dezoito entrevistados católicos, dez possuíam enfrentamento focalizado no problema, enquanto dos doze evangélicos, em cinco predominava o comportamento focalizado no problema. Contudo, o baixo $n$ da amostra impossibilitou desdobramentos dessa análise.

Todos os entrevistados avaliados como mal adaptados relataram a ocorrência de mudança em relação à vida profissional após a amputação e referiram entristecimento. Além disso, existiam outros fatores de estresse. Para um dos entrevistados avaliado como mal adaptado a estratégia de enfrentamento majoritária estava focalizada no problema e, para três entrevistados, estava focalizada na busca por práticas religiosas/pensamento fantasioso.

\section{DISCUSSÃO}

A perda de um membro pode desencadear várias mudanças na rotina do indivíduo ${ }^{11}$. Os dados obtidos no presente estudo apontam para um aumento da dependência, maior permanência em casa, abandono de atividades e emprego, o que faz com que a vida social seja reduzida. Alguns pacientes afirmaram a existência de um sentimento de ter "parado a vida" para cuidar da saúde.

É necessário atendimento psicológico ao paciente com questões emocionais que envolvem 
a doença ${ }^{3}$. Esse estudo possibilitou observar que a população de amputados estava sem cobertura de atendimento psicológico, talvez por existirem baixa compreensão do impacto da amputação para o indivíduo e por ter poucos psicólogos lotados no hospital campo deste estudo, considerando-se a quantidade de pacientes.

A maior parte das amputações desse estudo foi realizada nos membros inferiores incompletos, sendo a área de amputação mais atingida à região dos dedos dos pés, seguida por antepé, o que vai ao encontro a outro estudo realizado ${ }^{8}$ que concluiu ter sido mais frequente amputações nos dedos do pé, seguido por antepé e pé (18\%). Mesmo não sendo uma amputação de um membro completo foram relatados muitos desconfortos, como a sensação do membro fantasma ${ }^{9}$.

A patologia diabetes foi a mais frequente, representando $41,9 \%$ do diagnóstico dos pacientes amputados. Um estudo realizado aponta que essa patologia foi prevalente para $64 \%$ dos pacientes ${ }^{8}$. Tais achados confirmam ser o diabetes um dos principais problemas de saúde de pacientes que sofrem esse tipo de intervenção cirúrgica.

No presente estudo todos os pacientes com doenças obstrutivas eram ou foram tabagistas, o que vai de encontro a outro estudo, cujo tabagismo também foi prevalente $(59 \%)$ nos casos de amputação devido a doenças vasculares ${ }^{7}$. O tabagismo é um fator de risco importante, e mesmo sabendo disso, grande parte dos pacientes tabagistas relataram o sentimento de impotência para abandonar o cigarro, sendo este mais um fator de sofrimento para o paciente.

Considerando apenas os pacientes amputados por traumas em um estudo ${ }^{5}, 96,3 \%$ eram do sexo masculino. Em outro estudo realizado ${ }^{20}$, o gênero masculino prevaleceu entre os entrevistados, tendo a causa acidental como a mais frequente. No presente estudo os três pacientes que sofreram amputação por trauma eram do sexo masculino. Tais dados apontam para uma maior incidência de amputações por trauma em sujeitos do sexo masculino, o que pode sugerir que esta ocorrência seja mais bem explicada pelo comportamento dicotômico socialmente esperado e apresentado por homens e mulheres, que acaba por refletir na exposição mais frequente à maiores contingências passiveis de trauma por homens.

A prevalência do enfrentamento focalizado no problema pode ter sido influenciada pela visão positiva da amputação. Muitos dos entrevistados consideraram a amputação como a eliminação do perigo de perder a vida, uma possibilidade para o fim da internação e uma forma de manejar a situação estressora que, neste caso, seria a dor.
Um estudo ${ }^{6}$ concluiu que o apoio recebido pelos pacientes foi um preditor para o potencial de risco ou proteção para o desenvolvimento de depressão e ansiedade, o que também foi observado neste estudo, pois todos os entrevistados avaliados como mal adaptados relataram insatisfação quanto ao suporte operacional e destes, três também apontaram ser disfuncional o suporte emocional recebido.

Em um estudo ${ }^{15}$ sobre o processo de enfrentamento no pós-tratamento do câncer de mama, as estratégias focalizadas na emoção foram a menos enfatizadas pelos respondentes, assim como na investigação de outros estudiosos ${ }^{16}$ sobre a influencia na utilização de estratégias de enfrentamento pós transplante cardíaco, cujo resultado apontou uma maior utilização do enfrentamento focalizado na resolução do problema e uma menor utilização do enfrentamento focalizado na emoção.

Um estudo ${ }^{6}$ investigou durante a hospitalização, o estado emocional e o enfrentamento de pacientes submetidos à amputação no período pós-cirúrgico e concluiu com o uso da EMEP que a estratégia mais utilizada pelos pacientes foi à busca de práticas religiosas/pensamento fantasioso. O enfrentamento focalizado no problema também apresentou score elevado. As estratégias focalizadas na emoção e a busca por suporte social tiveram scores inferiores. Após seis meses da amputação as estratégias focalizadas na emoção foram a menos utilizadas pelos sujeitos.

No presente estudo as estratégias de enfrentamento focalizadas no problema e na prática religiosa/pensamento fantasioso foram mais frequentes, apenas para um dos entrevistados a busca de suporte social foi prevalente, não tendo nenhum entrevistado utilizado a estratégia de enfrentamento focalizada na emoção. Tal conclusão está de acordo com outros estudos ${ }^{6,15 e 16}$, onde a estratégia de enfrentamento focalizada na emoção também foi menos utilizada. Sugere-se esta ocorrência, no presente estudo, devido a maior parte dos pacientes terem recebido informações antes da amputação que permitiram haver um preparo emocional.

O paciente pode tentar mascarar a dor vivida para poupar a família. Existe a preocupação em relação à dependência, o que favorece o aparecimento de sentimentos de inferioridade e baixa autoestima $^{3}$. O aumento da dependência foi um dos desconfortos relatados por 12 dos entrevistados e mostrou-se associada à mudança social, pois estes afirmaram que passaram a sair menos.

No presente estudo foi verificado que $51,6 \%$ dos entrevistados eram aposentados ou 
recebiam auxílio pelo INSS e 12,9\% estavam tentando aposentar-se. Não houve relação estatisticamente significativa entre a adaptação e a aposentadoria. Dos entrevistados aposentados, oito já estavam aposentados antes da amputação devido à idade. Apenas treze dos entrevistados não relataram preocupação referente à vida profissional.

Não foram encontradas pesquisas publicadas no Brasil que discutem a relação entre o modo de enfrentamento e a adaptação do paciente na amputação por trauma e por doença. Sugere-se que distinguir o tipo de estratégia de enfrentamento mais utilizada por pacientes adaptados possibilitaria ao profissional psicólogo o aprendizado de um novo repertório de habilidades capazes de maximizar a independência do indivíduo amputado.

Observa-se assim, a necessidade da

\section{REFERÊNCIAS}

1. Montiel A, Vargas MAO, Leal SMC. Caracterização de pessoas submetidas à amputação. Enfermagem em foco. 2012; 3(4):169-173.

2. Magalhães MGM \& Santos GS. Amputação de membros inferiores. FacRedentor. 2012; 1-14. Disponível em: http:// arquivos.5gsistemas.com.br/PosRedentor/arquivos/conteudo 542af5947d17a.pdf. Acesso em: 23 de fevereiro de 2018.

3. Chini GCO, Boemer MR. A amputação na percepção de quem a vivencia: Um estudo sob a ótica fenomenológica. Rev Latino-am enfermagem. 2007; 15(2). Disponível em: http://www.scielo.br/scielo.php?script=sci_arttext\&pi04-11692007000200021\&lng=en\&nrm=iso\&tlng=pt. Acesso em: 04 de Junho de 2018.

4. Umburanas RC, Dubiela A, Pereira CS, Novak VC. Amputação de membro inferior: perfil dos pacientes do serviço de reabilitação física da Unicentro - projeto órtese e prótese. In: XII Encontro Latino Americano de Iniciação Científica; IX Encontro Latino Americano de Pós-graduação; III Encontro Latino Americano de Iniciação Científica Júnior. São José dos Campos. 2009. Disponível em: http://www.inicepg.univap.br/ cd/INIC_2009/anais/arquivos/0643_0982_01.pdf. Acesso em: 23 de dezembro de 2017.

5. Agne JE, Cassol CM, Bataglion D, Ferreira FV. Identificação das causas de amputações de membros no hospital universitário de Santa Maria. Saúde. 2004; 30(1-2): 84-89.

6. Gabarra LM. Estados emocionais, formas de enfrentamento, rede de apoio e adaptação psicossocial em pacientes amputados (Tese de doutorado). Florianópolis: Universidade Federal de Santa Catarina; 2010. 226p.

7. Seidel AC, Nagata AK, Almeida HC, Bonomo M. Epistemologia sobre amputação e desbridamentos de membros inferiores realizados no Hospital Universitário de Maringá. J. Vasc. Bras. 2008; 7(4): 308-315.

8. Miliole R, Vargas MAO, Leal SMC, Montiel AA. Qualidade de vida em pacientes submetidos à amputação. RevEnfermUFSM. 2012; 2(2): 311-319.

9. Chini GCO, Boemer MR. As facetas da amputação - Uma primeira aproximação. RevBrasEnferm. 2002; 55(2): 217-222.

10. Galván GB, Amiralian MLTM. Corpo e identidade: reflexões acerca da vivência de amputação. EstudPsicol. 2009; 26(3): 391-98.

11. Rudnicki T, Sanchez MM (Orgs). Psicologia da saúde: A prática da Terapia Cognitivo-Comportamental em hospital geral. Novo Hamburgo: Sinopsys. 2014. Disponível em: https://www. realização de outros estudos com uma amostra maior, com controle do tipo de amputação (trauma e doença) e com acompanhamento longitudinal dos sujeitos.

\section{CONCLUSÃO}

A análise dos achados sugere uma não associação entre estratégias de enfrentamento e adaptação. Tal fato, contudo, não significa que tais associações não existam e que seu estudo e compreensão não sejam relevantes para a atuação do psicólogo na atenção pré ou pós-cirúrgica ao paciente amputado.

Não foi possível concluir se não existe diferença quanto à estratégia de enfrentamento de pacientes amputados por trauma ou por doença ou se a amostra reduzida prejudicou a análise.

sinopsyseditora.com.br/upload/produtos_pdf/195.pdf. Acesso em: 23 de dezembro de 2017.

12. Silveira LMC, Ribeiro VMB. Grupo de adesão ao tratamento: espaço de "ensinagem" para profissionais de saúde e pacientes. Interface - Comunic., Saúde, Educ. 2005; 9(16): 91-104

13. Straub RO. Psicologia da saúde. Trad. Costa RC; Porto Alegre: Artmed; 2005.151-189.

14. Almeida FF. Análise das variáveis psicossociais relacionadas à experiência dolorosa entre pacientes cirúrgicos (Dissertação de mestrado). Brasília: Universidade de Brasília; 2011. 112p.

15. Silva G. Processo de enfrentamento no pós-tratamento do câncer de mama. (Dissertação de Mestrado). São Paulo: Faculdade de Filosofia, Ciências e Letras de Ribeirão Preto/ USP; 2005. 151p.

16. Pfeifer PM, Ruschel PP. Preparo psicológico: a influência na utilização de estratégias de enfrentamento pós-transplante cardíaco. Rev. SHPH. 2013; 16(2): 153-163.

17. Seidl EMF, Tróccoli BT. Desenvolvimento de escala para avaliação do suporte social em HIVIAIDS. Psicologia: Teoria e Pesquisa. 2006; 22(3): 317-326.

18. Organização Mundial de Saúde. Classificação de transtornos mentais e de comportamento da: CID-10. Porto Alegre: Artmed; 2008; 146-149.

19. Seidl EMF, Tróccoli BT, Zannon CMLC. Análise fatorial de uma medida de estratégias de enfrentamento. Psicologia: Teoria e Pesquisa. 2001; 17(3): 225-234.

20. Araújo DMS, Carvalho DO. Qualidade de vida e funcionalidade dos amputados em membros inferiores do hospital Santa Marcelina. 2016. Disponível em: http://repositorio.saolucas.edu.br:8080/xmlui/bitstream/handle/123456789/2030/ Daise $\% 20$ Maria\%20Siqueira\%20de\%20Ara\%C3\%BAjo,\%20 D\%C3\%A9bora\%200mido\%20de\%20Carvalho\%20-\%20 Qualidade $\% 20$ de $\% 20$ vida\%20e\%20funcionalidade $\% 20$ dos $\% 20$ amputados $\% 20$ em $\% 20$ membros $\% 20$ inferiores $\% 20$ do $\% 20$ Hospital\%20Santa $\% 20$ Marcelina.pdf?sequence=1. Acesso em: 23 de dezembro de 2017

\section{CORRESPONDÊNCIA}

Ana Paula da Silva Vasques Oliveira

Quadra 21 Casa 91 Setor Leste Gama DF - CEP: 72460-210

E-mail: psi.paula.vasques@hotmail.com 